\title{
A comparison of the LPIM-COSMIC F2 peak parameters determinations against the IRI(CCIR)
}

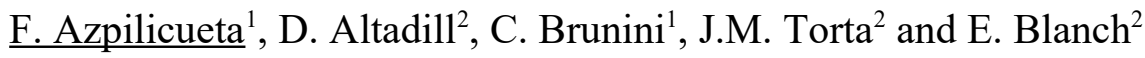 \\ ${ }^{1}$ GESA - Facultad de Cs. Astronómica y Geofísicas - Universidad Nacional de La Plata- \\ CONICET, Paseo del bosque s/n, La Plata, Argentina - azpi@,fcaglp.unlp.edu.ar \\ ${ }^{2}$ Observatori de l'Ebre, (OE) CSIC - Univ. Ramon Llull, Roquetes (Spain) \\ daltadill@obsebre.es \\ 7
}

\section{Abstract}

During the last decade the amount of ionosphere measurements, from ground and space born sources, has substantially increased along with the development of high end processing systems. This constitutes a perfect scenario for the International Reference Ionosphere (IRI) to assimilate this huge new database and to satisfy the new needs of the aeronomic community: an IRI with meteorological capability.

The first step before the implementation of an assimilative procedure is to validate the new data. This work intends to contribute in this direction by studying the differences (systematic and statistical) between the F2 peak parameters $(\mathrm{hmF} 2$ and $\mathrm{NmF} 2)$ predicted by the IRI(CCIR) and those obtained from the electron density profiles computed with LPIM-COSMIC/Formosat3 (LPIM-C/F3) technique. The analyzed period extends from January 2007 to October 2012, thus covering all the different seasonal Sun-Earth configurations and a range of solar activity going from low to mid-high level.

The analysis shows that there is no significant systematic bias between the IRI and LPIM-C/F3 values on both parameters. The obtained differences are comparable to those found between IRI and other models and data sources. In addition a correlation with the solar activity level is observed. The analysis performed is also helpful to study and asses the potentiality of the meteorological information contained in the LPIM-C/F3 by analyzing the standard deviation of the differences. This extra information could represent the key element to improve the IRI predicting capabilities.

Keywords: Ionosphere; IRI; LPIM; COSMIC/F3 mission, NmF2 and hmF2.

\section{Corresponding author}

Francisco Azpilicueta

E-mail address: azpi@fcaglp.unlp.edu.ar

Phone: 00542214236593 (ext. 154). Fax: 00542214236591

\section{Introduction}

The International Reference Ionosphere model (IRI) is the indisputable international standard when referring to scientific studies or technological applications related to the upper 
layers of the terrestrial atmosphere. Since the first developments in the late ' 60 produced by the Committee on Space Research (COSPAR) and the International Union of Radio Science (URSI), IRI has been permanently updated on its mathematical formulation thanks to the better knowledge provided by new techniques, measurements and databases contributing to the model: IRI-78, IRI-85, IRI-1990, IRI-2000, IRI-2007. For a detailed description of the evolution and current state of IRI see (Bilitza et al., 2011). During the last decade, the amount of ionospheric measurement available from ground and space borne instruments has increased substantially. The availability of new data sources along with new high-end data processing and communication systems give the perfect scenario for IRI to satisfy the new needs of the aeronomic community: an IRI capable to meteorologically predict the ionospheric (Bilitza et al., 2011). With this respect it is important to mention the remarkable effort carry on by the scientific staff of GIRO (Global Ionospheric Radio Observatory, http://giro.uml.edu/) (Reinisch and Galkin, 2011; Galkin et al., 2012).

The problem of switching from a climatological model to forecasting time-space model involves several issues. One of them is the assimilation of as much as data of as much as different data sources as possible. This latter is a key point because IRI is mainly based on data from ground ionosondes that have reasonable continental coverage. However, ionosonde data reports huge data gaps at the oceans and the Northern hemisphere is better covered than the Southern one. This problem can be overcome using data from satellites that systematically scan the ionosphere over oceans and continents. An outstanding example of this is the COSMIC/Formosat3 (C/F3) satellite mission which constitutes the data source of our work. Section 2 contains a summary with the basic information about the mission.

The specialized literature contains contributions with different assimilating approaches of ionosphere data from satellite missions. One that is widely used consists on using raw TEC (total electron content) measurements along the ray path between the GPS and the C/F3 satellites (Yue et al, 2012; Komjathy et al., 2010; Bust et al., 2004; Thompson et al., 2009). A different approach consists on making use of the RO electron density profiles (RO-EDP) provided by the C/F3 mission staff to estimate, via a Least Squares adjustment, the driving parameters of a profiler (Hoque and Jakowski, 2012), or the coefficients of an empirical orthogonal function expansion after preprocessing and gridding the data (Zhang et al., 2014). In this work we estimated the parameters of the F2 layer peak using the C/F3 RO-EDP as the input data. And for doing this we applied a modified version of the LPIM (Brunini et al., 2013). The processing with LPIM seeks two main objectives: i) data cleaning, to identify and eliminate those RO-EDP or ED punctual values that do not fulfill the quality criteria; ii) 
transforming the RO-EPD data into F2 peak parameters equivalent to those used by the IRI to anchor the profiles. Section 3 contains a summary of the main characteristics of LPIM and its implementation.

As with every assimilation procedure, the first step is to validate the new data that will be included in the processing scheme, so to analyze and assess the a-priori differences between the model and the new data. Understanding these differences can serve to prevent misleading results after the assimilation and to gain some insight about the potential enhancement. Then the last step in an assimilation work consists on comparing the results given by the assimilated model (a-posteriori) with an independent data source so to assess the improvements. Many articles related to assimilation/validation of space borne ionosphere data have been published during the last years. This is particularly evident with works related to RO data.

An outstanding work of a-posteriori validation is (Yue et al., 2012). In this work the authors assimilate TEC data from ground-based and several RO satellite missions (including C/F3) into IRI 2007, for the 2002-2011 time period. Then they compare the assimilated model with independent data: IGS global ionospheric maps, Poker Flat Incoherent Scatter Radar and a global network of ionosondes. One of the conclusions the authors found is the drastic positive impact the C/F3 data produce. Another interesting and recent article of this kind is (Zhang et al., 2013). In this work hmF2 maps computed based on data from an extended network of ionosondes are compared with the equivalent C/F3 and IRI model predicted maps. The comparisons show a global mean difference between the ionosonde and the C/F3 maps of $11.6 \mathrm{~km}(\sim 5.5 \%)$ for 2008 and $-2.8 \mathrm{~km}(\sim 9.5 \%)$ for 2012 . For the comparison between IRI and $\mathrm{C} / \mathrm{F} 3$ the authors found global mean differences of about $23.37 \mathrm{~km}(\sim 10.6 \%)$ for 2008 and $9.5 \mathrm{~km}(\sim 4.4 \%)$ for 2012 .

This work aims to contribute in the a-priori validating direction by studying the systematic and statistical differences observed between the F2 peak parameters estimated from the $\mathrm{C} / \mathrm{F} 3$ electron density data files and predictions computed by the IRI current formulation.

\section{COSMIC/Formosat 3 mission}

The dataset used in this research is composed by EDP derived from the GPS receivers flying onboard the COSMIC/Formosat3 (C/F3; Constellation Observing System for Meteorology, according to the USA nomenclature / Formosa Satellite Mission 3, according to the Taiwanese nomenclature) satellites in the framework of a collaborative project of the 
National Space Organization (NSPO) of Taiwan and the University Corporation for Atmospheric Research (UCAR) of the USA.

The constellation is composed of six micro-satellites that were launched on April 2006, into circular orbits with an altitude of $512 \mathrm{~km}$ and an inclination of $72^{\circ}$. During the first 17 months after the launch the satellites were gradually moved into their final orbits at $\sim 800 \mathrm{~km}$ of height, with a separation of $30^{\circ}$ in longitude between the nodes of neighboring orbital planes. For more details see (Anthes, 2008)

The primary payloads of the satellites are GPS radio occultation receivers, which allow computing profiles of atmospheric refractivity. Figure 1 contains a schematic representation of a radio occultation ( $\mathrm{RO}$ ) event between a C/F3 and a GPS satellite. These refractivity profiles can be later used to derive electron density profiles in the ionosphere by means of an Abel's inversion technique producing data files the vertical distribution of the electron density (Yue et al., 2010). The dataset used in this work was downloaded from the Data Analysis and Archival Center (CDAAC) database (http://cdaac-www.cosmic.ucar.edu/cdaac/products.html) at the UCAR, and consist of the EDP files computed by the C/F3 mission team for each RO event during the January 2007 - October 2012 period.

Figure 1: schematic representation of a radio ocultation event between a C/F3 and a GPS satellite.

\section{LPIM and RO data: $N_{m} F 2$ and $h_{m} F 2$ estimation algorithm}

As was already mentioned in Section 1, in this work we have used a modified version of the LPIM (Brunini et al., 2013) with two main objectives: i) to identify and eliminate those RO-EDP or ED punctual values that do not fulfill the quality criteria, in other words data cleaning; ii) transforming the RO-EPD data into F2 peak parameters equivalent to those used by the IRI to anchor the vertical profiles. The technique used in this work to retrieve the F2 peak parameters is based on the LPIM model (Brunini et al., 2013). LPIM represents the vertical distribution of the electron density by two different functions depending on the height. For the bottom-side (heights below $h_{m} F 2$ ) the function is the result of combining three $\alpha$-Chapman layers (corresponding to the E, F1 and the lower part of the F2 layer). For the topside (heights above $h_{m} F 2$ ) the vertical distribution is represented by a vary-Chapman function. The parameters of all the functions involved are related in such a way that the complete vertical profile (for heights between 60 to $1000 \mathrm{~km}$ ) is described by the three main 
parameters of the F2 peak: $N_{m} F 2, h_{m} F 2$ and $H F 2$ (scale height). LPIM apply the ITU-R (1997) recommendations for the bottom-side parameters and it evaluates the top-side parameters (the $\mathrm{O}^{+}$to $\mathrm{H}^{+}$transition height, scale height, and the shape parameter) according to Meza et al. (2008).

In summary, for a given point within the ionosphere and Universal Time, the LPIM electron density is given by a function that depends on the three F2 peak parameters. This formulation provides the estimation of $N_{m} F 2, h_{m} F 2$ and $H F 2$ (along with their estimated errors) by fitting the LPIM profile to RO electron density determinations. The fitting process is performed by LPIM using a re-weighted Least Squares procedure. In the first iteration all the RO determinations receive the same weights and then, these are modified according to the difference between the RO determination and the estimation given by the model in the previous iteration. The weighting criterion is based on a 'bisquare' function (Huber, 1981). The process is stopped when the changes in the solution is negligible. For simplicity the result of C/F3 EDP profiles with LPIM will be called LPIM-C/F3 data.

\section{Pre-processing considerations on the database and the time period}

We will focus on the climatological performance of the IRI in relation to the LPIM-C/F3 data estimation. That is why the analyses in the following sections will be based on monthly-averages. Thus, it is important to control the evolution of the number of RO events per month for the almost five years of data (Jan 2007 to Oct 2012). Fig.2 shows the global number of RO (black dots) and the number of events in the Northern (red dots) and in the Southern (blue dots) modip (modified dipolar latitude) Hemispheres. For details about modip coordinate system see (Rawer 1984; Azpilicueta et al., 2006). It is clear that the number of events decreases (a factor of 3) with time mainly due to aging of the instruments (Yue et al., 2012). The figure also shows that this decrease affects similarly both hemispheres, that is why we can discard inter-hemisphere artifact differences due to sampling reasons.

Figure 2: evolution of number of RO events for the data set used in the work. The black circles correspond to the global number of events, the red dots to the RO events in the Northern and blue ones to the Southern modip hemisphere.

The maps of Fig. 3 represent the distribution of the RO events in the Local Time-modip coordinate system for January 2007 (left) and January 2012 (right). Note that the Sun is always over the central meridian in this system and that Sunrise and Sunset are approximately at $-6+6$ hours respectively. These maps show the uneven distribution of the events and, at the 
same time, the larger data gaps in the density of events observed in 2012 in relation to 2007 which is the result of the decrease in the number of RO events.

Fagure 3: spatial distribution of RO events in the LT-modip coordinate system for January 2007 (left) and January 2012 (right). The central meridian is the sub-solar point meridian.

Another factor to take into account in the analysis is the solar activity level during the analyzed period. Figure 4 shows the behavior of the F10.7 index (a proxy for the solar activity) between 2000 and 2012. The squares correspond to the time interval 2007-2012 indicating that this work comprises period from low-to-mid level solar activity. As it will be seen, the results that follow are directly conditioned by this element.

Figure 4: temporal behavior of the F10.7 index for the 2000-2015 period. The squares correspond to the analyzed period.

\section{Reconstructing the ionospheric main structures through the LPIM-C/F3 data}

Figure 5 shows maps (in LT-modip coordinate system) of the mean global behavior of the $\mathrm{NmF} 2$ parameter (in 1.e10 electrons $/ \mathrm{m}^{3}$ ) obtained with the LPIM-C/F3 technique. The left map corresponds to March 2007 and the right one to March 2012. The individual inspection of each map reveals the expected double peak of the Ionospheric Equatorial Anomaly (IEA also known as Appleton Anomaly), with the valley following the magnetic Equator in between. The comparison between the two maps shows a clear increase on the anomaly mean ionization level and on the spatial extension of the peaks for 2012, following the Solar cycle (see Fig.4).

Figure 5: LT-modip representation of the $\mathrm{NmF} 2$ (in $1 . \mathrm{e} 10$ electrons $/ \mathrm{m}^{3}$ ) obtained by LPIM-C/F3 for March 2007 (left) and 2012 (right).

Figure 6 is similar to Fig. 5 but for hmF2. The left map correspond to March 2007 and the right one to March 2012. These maps show the expected behavior for the F2 peak height: a region with the higher altitudes centered at the magnetic Equator. It can be guessed a double peak structure in the hmF2 near noontime following the IEA and better shown at low solar activity. Also interesting is the observed highest altitudes region at the end of the anomaly, after sunset in the equatorial regions which correlates with the pre-reversal enhancement (PRE) phenomenon (Kelley et al., 2009). 
Figure 6: LT-modip representation of the $\mathrm{hmF} 2$ (in $\mathrm{km}$ ) obtained with LPIM-C/F3 for March 2007 (left) and 2012 (right).

The previous figures and analysis are quite useful to conclude that the information obtained by the LPIM-C/F3 technique is qualitatively significant. The comparisons contained in the following sections will add information about the quantitative dimension of the problem.

\section{Procedure and results}

As it was already discussed in Section 1, this work aims to assess the quality of the F2 peak parameters that can be obtained from LPIM-C/F3 profiles. Then for each RO-EDP processed, we have computed the corresponding values for $\mathrm{NmF} 2$ and $\mathrm{hmF} 2$ predicted by IRI so to calculate the differences $\Delta \mathrm{NmF} 2$ and $\Delta \mathrm{hmF} 2$ - LPIM-C/3 minus IRI - for both parameters. As an example of this Fig. 7 shows the geographical behavior of the monthly mean of $\Delta \mathrm{NmF} 2$ and $\Delta \mathrm{hmF} 2$ (left and right panel respectively) both for March 2012. Bluish color means that IRI predicts higher values than LPIM-C/3 estimates and reddish color means the opposite. The region with significant differences corresponds to the IEA, as expected. The $\Delta \mathrm{NmF} 2$ map shows that LPIM-C/F3 gives systematically higher values that IRI for the valley between the peaks and also at the outer edges of the peaks. The main differences observed for $\mathrm{hmF} 2$ locate near the peaks of the IEA and near the region of the PRE.

Figure 7: LPIM-C/F3 - IRI in the LT-modip system for March 2012. Left: $\Delta \mathrm{NmF} 2$ and (in 1.e10 electrons $/ \mathrm{m}^{3}$ ), right: $\Delta \mathrm{hmF} 2$ (in $\mathrm{km}$ ).

The next step for gaining a global view of the general agreement (or disagreement) between LPIM-C/F3 and IRI consisted on computing global monthly mean and standard deviation (SD) of $\Delta \mathrm{NmF} 2$ and $\Delta \mathrm{hmF} 2$ for each month of the complete. In order to detect any possible asymmetry between hemispheres, three sets were computed for each month: one corresponding to the global mean, the second for those $\mathrm{RO}$ events in the Northern hemisphere and the third one with the RO events in the Southern hemisphere. Fig. 8a shows the temporal evolution of the means and Fig.9a the SDs of the of $\Delta \mathrm{NmF}$, where the black curves correspond to the global average, the red ones to the Northern hemisphere and the blue curves to the Southern hemisphere.

Figure 8: Left: temporal evolution of the monthly means of $\Delta \mathrm{NmF} 2$ (in 1.e10 electrons $/ \mathrm{m}^{3}$ ). Right: monthly means of $\Delta \mathrm{NmF} 2$ against monthly mean of $\mathrm{F} 10.7$ (in s.f.u.). 
Figure 9: Left: temporal evolution of the monthly SDs of $\Delta \mathrm{NmF} 2$ (in $1 . e 10$ electrons $/ \mathrm{m}^{3}$ ). Right: monthly SDs of $\Delta \mathrm{NmF} 2$ against monthly mean F10.7 (in s.f.u).

Figure $8 \mathrm{~b}(9 \mathrm{~b})$ shows the monthly means (SD) of $\triangle \mathrm{NmF} 2$ plotted against the corresponding monthly means of F10.7. As it will appear in the following section the correlations with the monthly F10.7 help to understand the results. Figures 10 and 11 are similar to the two previous but for $\Delta \mathrm{hmF}$.

Figure 10: Left: temporal evolution of the monthly means of $\Delta \mathrm{hmF} 2$ (in $\mathrm{km}$ ). Right: monthly means of $\Delta \mathrm{hmF} 2$ against monthly means of F10.7.

Figure 11: Left: temporal evolution of the monthly SDs of $\Delta \mathrm{hmF} 2$ (in km). Right: monthly SDs of $\Delta \mathrm{hmF} 2$ against monthly means of F10.7.

\section{Analysis and conclusions}

Before proceeding any further, it's convenient to clarify two main general concepts necessary for the following analysis. In the frame of this work the monthly means are associated with the climatologic agreement between LPIM-C/F3 and IRI. In other words to the agreement between the average state of the ionosphere predicted by IRI with respect to the average state reflected in the LPIM-C/F3 data for a particular month.

As LPIM-C/F3 data for a RO event is the result of processing RO measurements from a particular moment, it is reasonable to assume that LPIM-C/F3 contains a meteorological component in addition to the meteorological one. In the scheme of our work this metereological information is absorbed by the monthly SDs are a measure of the deviation of the "current state" (sensed by LPIM-C/F3) of the ionosphere from the mean (climatological) configuration. From its mathematical formulation IRI; it is not expected IRI to reproduce the "current" ionospheric conditions. Thus the SDs can help to understand the potential additional information about the meteorological state of the ionosphere contained in the LPIM/C/F3 data.

The following paragraphs contain a detailed analysis of the main features observed in the previous figures discriminated by parameters $(\Delta \mathrm{NmF} 2$ and $\Delta \mathrm{hmF} 2)$ and by statistics (mean and SD).

$\triangle N m F 2$ means: the curves in Fig.(8a) present a minimum for 2009 and then starts an increasing trend following the solar activity. The series are negative until 2012, indicating a systematic discrepancy (IRI larger than LPIM-COSMIC) for the period. Overlapped to the trend there is an annual signal indicating an increase during Decembers (when the Sun is over 
the Southern hemisphere). The hemispheric series do not present significant differences, and they seem to follow the global series.

Another way for studying the effect of the Solar activity is by plotting the means and SDs against the monthly means of F10.7 index (Fig.8b). The $\triangle N m F 2$ and F10.7 means have a correlation coefficient of .60 indicating that the agreement clearly depends on the solar activity level. Additionally, the best agreement for the period analyzed is not reached for the period with lowest activity, but for a rather high one (F10.7 index of 120 s.f.u.). The hemispheric series do not present significant differences, and they seem to follow the global series quite well.

$\triangle N m F 2 S D s$ : the curves in Fig.(9a) clearly show a trend that follows the solar activity, and also present a combination of an annual and a semi-annual signal. Analyzing a particular year, the SDs at equinoxes are larger than in other seasons and June solstices SDs show the lowest value. It seems that the SD is always low (less dispersion and therefore better agreement) for June solstices. The SDs (Fig. 9b) are more affected by the Solar activity than the means with a correlation coefficient of 0.81 The hemispheric series do not present significant differences, and they seem to follow the global series.

$\Delta h m F 2$ means: Fig.(10a) shows a trend that follows the solar activity and presents negative values (IRI larger than LPIM-COSMIC) until 2012. It seems that the mean tends to be a bit closer to 0 for June solstices, although is not as clear for $\Delta \mathrm{hmF} 2$ as for $\triangle \mathrm{NmF} 2$. The correlation coefficient (Fig.10b) is 0.43 indicating that a dependency with solar activity level but weaker than the one found for $\Delta \mathrm{NmF} 2(.60)$.

hmF2 SDs: the curves in Fig.(11.a) clearly show a trend that follows the solar activity. It also presents an annual signal in phase opposition between the hemispheres: the SD is larger for local summer. The Southern hemisphere shows larger amplitudes indicating that this hemisphere is more complex than the other. Once again the SDs are more affected than the means by the Solar activity level with a correlation coefficient of 0.88 .

Although not shown here, the mean difference in the hmF2 observed between LPIM$\mathrm{C} / \mathrm{F} 3$ and IRI is compatible with the differences observed between LPIM-C/F3 and hmF2 model of the Ebro team (Altadill et al., 2013).

\section{Conclusions and further works}

After the analysis presented in the previous section the main conclusions that can be formulated based on this work are: 
a) There is an issue regarding the degradation of the space born instruments that causes a significant reduction on the number of RO events. The regional data gaps should also be taken into consideration.

b) The means of $\triangle \mathrm{NmF} 2$ observed (LPIM-C/F3 - IRI) are compatible with the differences between IRI and other models and data sources. For example in (Yue et al., 2013) the authors found a range interval for the $\triangle \mathrm{NmF} 2$ of approx. $501.10 / \mathrm{m} 3$ when comparing results after the assimilation process with IRI predicted values. In other words there is no clear evidence of a systematic bias between LPIM-C/F3 and IRI.

c) The means of $\Delta \mathrm{hmF} 2$ (climatologic issue) does not present any systematic difference with a neglecting variation range from -10 to $10 \mathrm{~km}$, completely compatible with the values given in (Altadill et al., 2013). This could be interpreted as a verification that LPIM-C/F3 F2 peak parameters precision is comparable within the expected errors of IRI and any other data source.

d) The solar activity level enlarges the means of both parameters but mainly of $\Delta \mathrm{NmF} 2$ (0.6 against 0.4 for $\Delta \mathrm{hmF} 2$ ).

e) The statistics most affected by the solar activity are the SDs.

Acknowledgments. The authors would like to acknowledge the whole COSMIC/Formosat3 team at the University Corporation for Atmospheric Research (UCAR), for processing the data and opening the data RO-EDP to the scientific community. We also want to thank two anonymous reviewers for their constructive comments and suggestions that have significantly enhanced our manuscript.

\section{References}

Altadill, D., Magdaleno, S., Torta, J. M., Blanch, E. Global empirical models of the density peak height and of the equivalent scale height for quiet conditions, Adv Space Res, 52, 1756-1769, 2013.

Anthes, R. A., Bernhardt, P. A., Chen, Y., Cucurul, L., Dymond, K. F., Ector, D., Healy, S. B., Ho, S.-P., Hunt, D. C., Kuo, Y.-H., Liu, H., Manning, K., Mccormick, C., Meehan ,T. K., Randel, W. J., Rocken, C., Schreiner, W. S., Sokolovskiy, S. V., Syndergaard, S., Thompson, D., Trenberth, K. E., Wee, T.-K., Yen, N. L., Zeng, Z. The COSMIC / FORMOSAT 3 mission - Early results. Bulletin of the American Meteorology Society, March 2008, 313 - 333, 2008.

Azpilicueta, F., Brunini, C., Radicella S.M. Global ionospheric maps from GPS observations using modip latitude, Adv Space Res, 36, 552-561, 2006.

Bilitza, D., McKinnell, L.-A., Reinisch, B., Fuller-Rowell, T. The International Reference Ionosphere (IRI) today and in the future. J. Geodesy, 85:909-920, DOI 10.1007/s00190-010-0427-x, 2011.

Brunini, C., Azpilicueta, F., Nava, B. A technique for routinely updating the ITU-R database using radio occultation electron density profiles. J Geod, 87, 813-823, doi: 10.1007/s00190-013-0648-x, 2013. 
Bust, G.S., Garner T.S., Gaussiran T.L. II, Ionospheric Data Assimilation Three-Dimensional (IDA3D): A global, multisensor, electron density specification algorithm, J. Geophys. Res., 109, A11312, doi:10.1029/2003JA010234, 2004.

Galkin I., Reinisch, B., Huang, X., Bilitza C. Assimilation of GIRO data into a real-time IRI, Radio Scie, 47, RS0L07, doi: 10.1029/2011RS004952, 2012.

Huber, P. J. Robust Statistics. New York: John Wiley and Sons, 1981.

Hoque, M. and Jakowski N., A new global model for the ionospheric F2 peak height for radio wave propagation, Ann. Geophys., 30, 797-809, doi:10.5194/angeo-30-797-2012, 2012.

ITU-R Recommendation ITU-R P.1239. ITU-R reference ionospheric characteristics. International Telecommunications Union, Radio Communication Sector, Geneva, 1997.

Kelley, M.C., Ilma, R.R., Crowley, G. On the origin of pre-reversal enhancement of the zonal equatorial electric field. Ann. Geophys., 27, 2053-2056, 2009.

Komjathy, A., B. Wilson, X. Pi, V. Akopian, M. Dumett, B. Iijima, O. Verkhoglyadova, and A. J. Mannucci, JPL/USC GAIM: On the impact of using COSMIC and groundbased GPS measurements to estimate ionospheric parameters, J. Geophys. Res., 115, A02307, doi:10.1029/2009JA014420, 2010.

Meza, A., Gularte Scarone, E., Brunini, C., Mosert, M. Analysis of a topside ionospheric model using GPS and ionosonde observables. Advances in Space Research, 42, 712-719, doi 10.1016/j.asr.2007.08.042, 2008.

Rawer, K. (Ed.). Encyclopedia of Physics, Geophysics III, Part VII, Springer-Verlag, 387-391, 1984.

Reinisch, B., Galkin, I., Global Ionospheric Radio Observatory (GIRO), Earth Planets Space, 63, 377-381, 2011.

Thompson, D. C., Scherliess, L., Sojka, J. J., Schunk, R.W. Plasmasphere and upper ionosphere contributions and corrections during the assimilation of GPS slant TEC, Radio Sci., 44, RS0A02, doi:10.1029/2008RS004016, 2009.

Yue, X., Schreiner, W. S., Lei, J., Sokolovskiy, S. V., Rocken, C., Hunt, D. C., Kuo, Y.-H. Error analysis of Abel retrieved electron density profiles from radio occultation measurements. Ann. Geophys., 28, 217-222, doi:10.5194/angeo-28-217-2010, 2010.

Yue, X., Schreiner W.S., Kuo Y-H., et al. (2012), Global 3-D ionospheric electron density reanalysis based on multisource data assimilation, J. Geophys. Res., 117, A09325, doi:10.1029/2012JA017968.

Zhang M-L., Liu, L., Wan W., Ning, B. An update global model of hmF2 from values estimated from ionosonde and COSMIC/FORMOSAT-3 radio occultation, Adv Space Res, 53, 395-402, 10.1016/j.asr.2013.11.053, 2014. 